\title{
Antibacterial, anti-inflammatory, and antioxidant effects of Yinzhihuang injection ${ }^{1}$
}

\author{
Juan Liu ${ }^{\mathrm{a}, *}$, Hong Qiu ${ }^{\mathrm{a}}$, Zhaorong Zhu ${ }^{\mathrm{a}}$ and Tangbin Zou ${ }^{\mathrm{b}}$ \\ ${ }^{a}$ Department of Veterinary Medicine, Southwest University, 160\# Xue Yuan Road, Rongchang 402460, \\ Chongqing, China \\ ${ }^{b}$ School of Public Health, Guangdong Medical College, Dongguan, Guangdong, China
}

\begin{abstract}
The Yinzhihuang injection, a traditional Chinese medicine, has been the recent target of increasing interest due to its anti-inflammatory properties. The molecular basis by which Yinzhihuang injection could cure Riemerella anatipestifer (RA) serositis in ducks is unclear. This study evaluated the antibacterial, anti-inflammatory and antioxidant effects of Yinzhihuang injection, using disease models of RA-induced infectious serositis in ducks and heptane-induced inflammation in mice and rats. The duck mortality rate was reduced from $60 \%$ to $20 \%$ and both the inflammatory response and histological damage were ameliorated by treatment with Yinzhihuang injection $(0.02 \mathrm{~g} / \mathrm{kg})$. Further studies indicated that superoxide dismutase (SOD), nitric oxide synthase (NOS), and inducible nitric oxide synthase (iNOS) were elevated while malondialdehyde (MDA), nitric oxide (NO) and RA growth were inhibited when the ducks were treated by Yinzhihuang injection. In addition, Yinzhihuang injection $(0.04 \mathrm{~g} / \mathrm{ml})$ effectively inhibited xylene-induced auricle swelling in mice, (demonstrating an inhibition rate of 35.21\%), egg albumen-induced paw metatarsus swelling in rats, (demonstrating an inhibition rate of $22.30 \%$ ), and agar-induced formation of granulation tissue. These results suggest that Yinzhihuang injection ameliorates RA-induced infectious serositis in ducks by modulation of inflammatory mediators and antioxidation.
\end{abstract}

Keywords: anti-infective, anti-inflammatory, Yinzhihuang, riemerella anatipestifer

\section{Introduction}

Inflammation is a natural defensive mechanism of the body toward a pathogenic organism, which is initiated to minimize damage to the body system. It may result in various ailments, such as bronchitis, arthritis deformans, colitis, gravis, artherosclerosis, and/or parasitic diseases [1-3]. Inflammatory responses caused by infectious and non-infectious external factors induce reactive oxygen and nitrogen species, which include hydrogen peroxide $\left(\mathrm{H}_{2} \mathrm{O}_{2}\right)$, nitric oxide (NO), and reactive intermediates such as hydroxyl radicals $(\mathrm{OH} \bullet)$, superoxide $\left(\mathrm{O}_{2}^{\bullet-}\right)$, and peroxynitrite $\left(\mathrm{ONOO}^{-}\right)[4,5]$.

Among many factors which induce inflammation, biological factors such as bacteria, viruses, and parasites are very common $[6,7]$. Upon attack by these pathogens, the immune system promotes phagocytic chemotaxis and induces phagocytosis at the infection site. Furthermore, phagocytes release

\footnotetext{
${ }^{1}$ Juan Liu and Zhaorong Zhu contributed equally to this work.

* Address for correspondence: Juan Liu, Department of Veterinary Medicine, Rongchang Campus, Southwest University, 160\# Xue Yuan Road, Rongchang 402460, Chongqing, China. Tel.: +86 1375282 7556; Fax: +86 0234675 1732; E-mail: liujuanb@163.com.
} 
inflammation mediators and oxyradicals (OFRs). Inflammation mediators stimulated the inducible nitric oxide synthase (iNOS) gene and produced considerable plenty of iNOS. With the assistance of Inos and NO, peroxynitrite (ONOO-) came into being and then ONOO- may oxidizes proteins [8]. Due to the limited amount of antioxygen in organisms, redundant OFR reacts with unsaturated fatty acids in the cytoplasm, eventually damaging the cell membrane and cell organs to induce the liberation of inflammation mediators and generate inflammation, thus forming a cycle of infection [9-11]. Currently available treatment options primarily consist of synthetic anti-inflammatory agents such as non-steroidal anti-inflammatory drugs (NSAIDs) $[12,13]$. However, synthetic drugs are associated with higher occurrences of side effects and may cause metabolic disorders [14-17]. Traditional Chinese medicines are widely used to treat inflammation, making their functional mechanisms a popular research topic worldwide [18].

Treatment with Yinzhihuang injection has been found to protect the body from acute liver injury induced by thioacetamide in mice. Yinzhihuang injection also demonstrated efficacy in the treatment of intrahepatic cholestasis of chronic viral hepatitis B. This represents a potential treatment option that requires further investigation into its clinical applications [19]. Yinzhihuang injection can significantly improve abnormal changes in $\mathrm{Na}^{+}-\mathrm{K}^{+}$-ATPase activity and morphological structures of brain tissue in neonatal rats with bilirubin-induced brain injuries. Abnormal morphological changes in the cerebral cortex were improved by varying degrees in rats from different therapeutic groups [20].The main checmical component of the Yinzhihuang injection is baicalin, a flavonoid glycoside obtained from the dried roots of Scutellariabaicalensis Georgi. Baicalin has demonstrated a wide range of pharmacologic effects, including heat-clearance and detoxification, increased urination, and increased urination. It is generally used to treat oxidative stress, $\mathrm{H}_{2} \mathrm{O}_{2}$-induced cytotoxicity, and collagen-induced arthritis [21-24]. Contemporary research has shown that baicalin has a positive effect on antibiosis functions [25-27].

In an S. aureus mastitis model of a mouse, baicalin reduced the concentration of proinflammatory cytokines, including TNF-alpha, IL-beta, and IL-6. It also inhibited NF-kappaB and p38 phosphorylation, and mRNA expression [28]. Baicalin improved the survival rate among mice with polymicrobial sepsis, and significantly reduced levels of proinflammatory cytokines by increasing the amount of anti-inflammatory cytokine in the blood and peritoneum. Simultaneously, baicalin increases the number of CD4+CD8+T lymphocytes and dendritic cells (DCs), and reduces the number of CD4+CD25+T cells [29]. Therefore, we chose to explore the effects of Yinzhihuang injection on RA-induced infectious serositis in ducks by modulation of inflammatory mediators and antioxidation.

\section{Materials and methods}

\subsection{Materials}

\subsubsection{Animals}

BALB/c mice (18-22 g), all inbred males 7-8 weeks old, were supplied by Chongqing Medical University Laboratory Animal Center (Chongqing, China). Male Wistar rats (180-220 g) 12-15 weekds of age were supplied by Luzhou Medical College Laboratory Animal Center (Luzhou, Sichuan, China). Domestic ducks four days old were supplied by Southwest University Laboratory Animal Center (Chongqing, China). Animals were allowed free access to their standard diet and sterile water in a restricted access room with controlled temperature $\left(22-26^{\circ} \mathrm{C}\right)$, humidity $(50-60 \%)$, and a 12-hour light/dark cycle in the Laboratory Animal Center of Southwest University (Rongchang, Chongqing, 
China). The experimental protocol was in accordance with the Guidelines for Animal Experiments of Southwest University and approved by the Institutional Authority for Laboratory Animal Care.

\subsubsection{Drugs and reagents}

Yinzhihuang injections $(0.1 \mathrm{~g} / \mathrm{mL})$ were purchased from Chengdu Kunhong Animal Pharmaceutical Co. Ltd (Chengdu, Sichuan, China). Florfenicol injections (containing 10\% florfenicol) were purchased from Sichuan Huaxi Animal Medicine Co. Ltd. Nutrient broth medium was obtained from Qingdao Hope Bio-Technogy Co. Ltd. (Qingdao, China). Superoxide dismutase (SOD), nitric oxide synthase (NOS), iNOS, malondialdehyde (MDA) content, and NO kits were purchased from Nanjing Jiancheng Bioengineering Institute Co. Ltd. (Nanjing, China). Griess reagent $(0.1 \%$ naphthylethylenediaminedihydrochloride and $1 \%$ sulfanilamide in $5 \%$ phosphoric acid) was obtained from Invitrogen (Grand Island, NY), and lipopolysaccharide (LPS) was obtained from Sigma, St. Louis, MO). Triton was obtained from Shanghai SangonBiological Engineering Technology \& Services Co. Ltd.

\subsection{Methods}

\subsubsection{Riemerella anatipestifer-induced iousserositis in ducks}

Inbred 4-day-old ducks were intramuscularly (i.m.) injected with $0.3 \mathrm{~mL}$ RA $\left(10^{9} \mathrm{CFU} / \mathrm{mL}, \mathrm{AF}\right.$ strain, from the Preventive Medicine Department, Southwest University) diluted in nutrient broth medium (Hopebio, Qingdao, China) to induce acute iousserositis. Ducks were randomly assigned to three groups of ten ducks each: Yinzhihuang, florfenicol, or control group. In the normal control group, eight ducks were each injected with $0.3 \mathrm{~mL}$ i.m. nutrient broth medium.

Three hours after contracting RA, the Yinzhihuang group was injected with $0.2 \mathrm{~mL} / \mathrm{kg}$ weight of Yinzhihuang $(0.1 \mathrm{~g} / \mathrm{mL})$ once each day for three days. The florfenicol group was injected with 0.1 $\mathrm{mL} / \mathrm{kg}$ weight florfenicol (10\%) once each day for three days.

\subsubsection{Measurement of SOD, NOS, iNOS, MDA, and NO using assay kits}

Ducks in each group were tracked, and deceased ducks were dissected to observe the heart, liver, and lungs. Blood samples were collected from the surviving ducks, centrifuged (503.1g for 5 minutes. two weeks after modeling, SOD, MDA, NO, NOS, and iNOS content was determined from the serum using assay kits from Nanjing Jiancheng Bioengineering Institute.

\subsubsection{Oxford cup determination of RA inhibition by Yinzhihuang injection}

A petri dish with a diameter of $6 \mathrm{~cm}$ was placed with $15 \mathrm{ml}$ of sterilized agar medium and then 0.1 $\mathrm{ml}$ of agar culture medium containing RA. And then dishes were incubated above $45-50^{\circ} \mathrm{C}$. After solidification, four Oxford cups were kept in each petri dish and Yinzhihuang injections of varying concentrations $(0.04,0.02$, and $0.01 \mathrm{~g} / \mathrm{mL})$ were added. The petri dishes were cultured at a temperature of $25^{\circ} \mathrm{C}$ for 24 hours, and the diameters of inhibition zones were determined for each concentration of Yinzhihuang injection. The procedure was repeated three times.

\subsubsection{Mouse auricle swelling induced by xylene}

Fifty healthy male BALB/c mice were randomly assigned to five groups, with ten mice in each group. Each group was treated with $0.04 \mathrm{~g} / \mathrm{kg}, 0.02 \mathrm{~g} / \mathrm{kg}$, and $0.01 \mathrm{~g} / \mathrm{kg}, 0.04 \mathrm{~g} / \mathrm{kg}$, or $0 \mathrm{~g} / \mathrm{kg}$ Yinzhihuang. The $0 \mathrm{~g} / \mathrm{kh}$ group was treated with $2 \%$ sodium salicylate as a control. All treatments consisted of intraperitoneal injections (i.p.) of $0.2 \mathrm{~mL} / 10 \mathrm{~g}$ weight once per day for three days. The left 
ear of each mouse was treated with endermosis $50 \mu \mathrm{x}$ xylol after the first treatment administration, and the mice were exterminated by cervical dislocation under ether anesthesia; and ears were dissected one hour after death. Identical parts of the ear piece were removed during all disections, with a diameter of $5 \mathrm{~mm}$ punch and weight. The degree of swelling (grams) and inhibition rate (\%) were calculated, considering the weight of the right ear piece as $100 \%$ [30].

Degree of swelling $(\mathrm{g})=$ left ear piece weight $(\mathrm{g})$ - right ear piece weight $(\mathrm{g})$

Inhibition rate $(\%)=$ (average weight of right ears - average weight of left ears) / average weight of right ears $\times 100 \%$

\subsubsection{Rat toe swelling induced by egg albumen}

Twenty healthy male Wistar rats were randomly divided into five groups of four mice each, and were then weighed and marked. Each group $(0.04 \mathrm{~g} / \mathrm{kg}$ Yinzhihuang, $0.02 \mathrm{~g} / \mathrm{kg}$ Yinzhihuang, $0.01 \mathrm{~g} / \mathrm{kg}$ Yinzhihuang, and a sodium salicylate group containing $2 \%$ sodium salicylate) were treated once per day with i.p. $2.5 \mathrm{ml} / \mathrm{kg}$ weight for three days. The $0 \mathrm{~g} / \mathrm{kg}$ Yinzhihuang group was treated with i.p. normal saline $(2.5 \mathrm{ml} / \mathrm{kg}$ weight). After administration of treatment, the right postpedes of rats were injected with $0.5 \mathrm{ml}$ fresh egg albumen (Chongqing, China). After injection, the size of the granuloma pouch was measured, and the inhibitory rate of intumesce was calculated.

Inhibition rate $(\%)=$ (average intumesce rate of left postpedes - average weight of right postpedes $)$ / average weight of left ears $\times 100 \%$.

\subsubsection{Agar granulation tissue hyperplasia in mice}

Fifty healthy male BALB/c mice were treated with subcutaneous injection to the back. The subcutaneous injection consisted of $0.5 \mathrm{ml}$ agar solution (2\%). Mice were randomly divided into five groups after 24 hours. The $0.04 \mathrm{~g} / \mathrm{kg}$ Yinzhihuang group, $0.02 \mathrm{~g} / \mathrm{kg}$ Yinzhihuang group, $0.01 \mathrm{~g} / \mathrm{kg}$ Yinzhihuang group and the $2 \%$ NaSA group were treated with i.p. $0.2 \mathrm{ml} / 10 \mathrm{~g}$ weight once per day for seven days. On the eighth day, the mice were executed and their granulomas were dissected. The granuloma inhibition rates were calculated as follows:

Granuloma inhibition rate $(\%)=$ (average weight of granuloma in group - average weight of group granuloma / average weight of granuloma IV $\times 100 \%$

\subsubsection{Isolation of $B A L B / c$ mice peritoneal macrophages}

Murine peritoneal macrophages were obtained from BALB/c mice four days after injection of $4 \%$ thioglycolate. On Giemsa-stained smear tests, the purity of the macrophages was $98 \%$. Next, $2-5 \times$ $10^{5}$ cells were plated in 96-well culture plates (NO assays) and cultured in RPMI 1640 medium (invitrogen) supplemented with $100 \mathrm{u} / \mathrm{ml}$ penicillin and $100 \mu \mathrm{g} / \mathrm{ml}$ streptomycin at $37^{\circ} \mathrm{C}$ under $5 \%$ $\mathrm{CO}_{2}$ atmosphere [31].

\subsubsection{Nitric oxide quantification of LPS-induced peritoneal macrophages from BALB/c mice}

Murine peritoneal macrophages were plated in 96-well culture plates, as described above, and were divided into five groups: $0.04 \mathrm{~g} / \mathrm{ml}$ Yinzhihuang with $1 \mu \mathrm{g} / \mathrm{ml}$ LPS, $0.02 \mathrm{~g} / \mathrm{ml}$ Yinzhihuang with 1 $\mu \mathrm{g} / \mathrm{ml}$ LPS, $0.01 \mathrm{~g} / \mathrm{ml}$ Yinzhihuang with $1 \mu \mathrm{g} / \mathrm{ml}$ LPS, $0 \mathrm{~g} / \mathrm{kg}$ Yinzhihuang with $1 \mu \mathrm{g} / \mathrm{ml} \mathrm{LPS}$, and healthy mice with PBS. The level of nitric oxide, as measured by nitrite production, was determined at 2, 6, 8, and 12 hours after treatment using the Griess reagent (Invitrogen). The absorbance at $550 \mathrm{~nm}$ was measured, and the nitrite concentration was determined by UV spectrophotometer using a curve calibrated on sodium nitrite standards (TU-1901, Purkinje General Instrument Co. Ltd., Beijing, China) [31]. 
Table 1

Influence on survival rate of RA-infected ducks

\begin{tabular}{llll}
\hline Group & Number of deaths & Mortality rate $(\%)$ & Survival rate $(\%)$ \\
\hline $0.02 \mathrm{~g} / \mathrm{kg}$ Yinzhihuang $(n=10)$ & 2 & $20^{* *}$ & 80 \\
Florfenicol $(n=10)$ & 2 & $20^{* *}$ & 80 \\
No drug $(n=10)$ & 6 & 60 & 40 \\
Healthy $(\mathrm{n}=8)$ & 0 & 0 & 100 \\
\hline \multicolumn{4}{r}{ Note: ${ }^{*} p<0.05$ or ${ }^{* *} p<0.01$ vs. the No drug group. }
\end{tabular}

\section{Statistical analysis}

Most data is presented as mean $\pm \mathrm{SD}$. The statistical significance $(p<0.05$ or $p<0.01)$ was evaluated by t-tests between two groups and one-way ANOVA with simultaneous multiple comparisons among different groups in the Statistical Analysis System SPSS 20.0.

\section{Results}

\subsection{Effects of Yinzhihuang injection on clinical symptoms and mortality rate of ducks with $R A$-induced infectious serositis}

After 4-5 hours of RA infection, ducks displayed symptoms of mental depression, prostrate form, closed eyes and lethargy. After 36 hours of RA infection, ducks began to die, demonstrating a $75 \%$ mortality rate over two weeks. Anatomy of the deceased ducks showed that most of them had developed fibrinous pericarditis, cellulose, and cellulose perihepatitis. The cure rate incurred by Yinzhihuang injection was $80 \%$, as shown in Table 1 .

\subsection{Effects of Yinzhihuang injection on SOD, NOS, and iNOS activity}

After 3 hours of RA infection with RA, Yinzhihuang injection was administered to ducks intramuscularly once each day for three days. Blood serum from ducks was obtained two weeks after RA infection. SOD activity in Yinzhihuang-treated ducks infected with RA was higher in the control and florfenicol groups $(p<0.01)$, as shown in Figure 1A. Compared to the control group, the activity of NOS and iNOS in the $8 \mathrm{mg} / \mathrm{kg}$ Yinzhihuang and florfenicol groups was higher $(p<0.01)$. The activity of iNOS in the $0.02 \mathrm{~g} / \mathrm{kg}$ Yinzhihuang group was lower than that in the florfenicol group $(p<0.05)$, as shown in Figure 1B.

\subsection{Effects of Yinzhihuang injection on MDA and NO in serum of ducks}

After two weeks of RA infection, MDA and NO increased in the model group, Yinzhihuang group, and florfenicol group. The MDA content in the Yinzhihuang group was lower than that of the control group $(p<0.01)$, but demonstrated no significant difference in comparison to the florfenicol group $(p>0.05$, as shown in Figure 1C. The NO content in the Yinzhihuang group was lower than that in the control and florfenicol groups $(p<0.01)$, as shown in Figure 1C. 


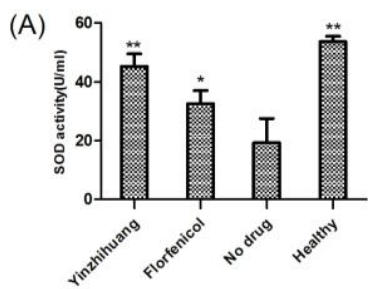

(B)
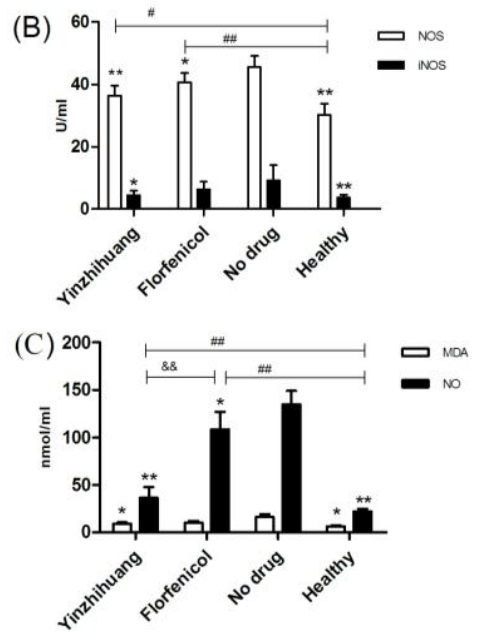

Fig. 1. Effects of $0.1 \mathrm{~g} / \mathrm{mL}$ Yinzhihuang injection on SOD, NOS, iNOS activities in duck serum. A: SOD; B: NOS and iNOS. C: MDA and NO. Serum samples were obtained from the ducks infected with RA for two weeks or ducks without RA infection (healthy group).

Notes: * and \# mean $P<0.05$ or **, \#\# and \&\& mean $P<0.01$

\subsection{In vitro anti-RA effects of Yinzhihuang injection}

The Yinzhihuang injection inhibited RA growth, as determined by the Oxford Cup method. Yinzhihuang injection of $0.04 \mathrm{~g} / \mathrm{ml}$ was particularly effective, as shown in Table 2.

Table 2

Influence on inhibition zone of Yinzhihuang injection on RA in vitro

\begin{tabular}{clll}
\hline & $1 \%$ Yinzhihuang & $2 \%$ Yinzhihuang & 4\%Yinzhihuang \\
\hline Inhibition zone $(\mathrm{mm})$ & $12.33 \pm 0.58$ & $16.77 \pm 0.25^{* *}$ & $21.33 \pm 0.58^{* *}$ \\
\hline Note: mean \pm S.D. (n= 4/each group). ${ }^{* *} P<0.01$ vs. the $1 \%$ Yinzhihuang group.
\end{tabular}

Table 3

Effect of Yinzhihuang injection on mouse auricle swelling induced by xylene

\begin{tabular}{llllll}
\hline Group & Drug & Dosage $(\mathrm{g} / \mathrm{kg})$ & Volume $(\mathrm{mL} / 10 \mathrm{~g})$ & Weight change $(\mathrm{g})$ & Inhibition rate $(\%)$ \\
\hline 1 & Yinzhihuang & 0.04 & 0.2 & $0.0046 \pm 0.0020 * * \#$ & 35.21 \\
2 & Yinzhihuang & 0.02 & 0.2 & $0.0061 \pm 0.0031 * \&$ & 14.08 \\
3 & Yinzhihuang & 0.01 & 0.2 & $0.0064 \pm 0.0050 * \&$ & 9.88 \\
4 & Yinzhihuang & 0 & 0.2 & $0.0071 \pm 0.0025$ & 0 \\
5 & $2 \%$ NaSA & 0.5 & 0.2 & $0.0062 \pm 0.0012 * \&$ & 12.68 \\
\hline
\end{tabular}

Note: weight changeisexpressed as mean \pm S.D. (n= 10/each group). ${ }^{*} p<0.05,{ }^{* *} p<0.01$ vs. Yinzhihuang $(0 \mathrm{~g} / \mathrm{kg}) ; \# p<0.05 \mathrm{vs.} 2 \% \mathrm{NaSA}$ group V; $\& p<0.05$ vs. $0.04 \mathrm{~g} / \mathrm{kg}$ Yinzhihuang. 


\subsection{Effect of Yinzhihuang injection on xylene-induced auricle swelling in mice}

Effect of Yinzhihuang injection on mouse auricle swelling varies with different concentrations of injection. The inhibition rate of swelling in the $0.02 \mathrm{~g} / \mathrm{kg}$ Yinzhihuang group was lower than that of the control group, as shown in Table 3.

\subsection{Effect of Yinzhihuang injection on egg albumen-induced rat postpedes metatarsus swelling}

The effect of Yinzhihuang injection on toe swelling in rats was most effective in the 0.04 and 0.02 $\mathrm{g} / \mathrm{kg}$ Yinzhihuang groups, which demonstrated inhibition rates of $22.30 \%$ and $21.03 \%$, respectively. The demonstrated inhibition rates of the $10 \% \mathrm{NaSA}$ and $0.01 \mathrm{~g} / \mathrm{kg}$ Yinzhihuang groups were less than in the 0.04 and $0.02 \mathrm{~g} / \mathrm{kg}$ Yinzhihuang groups, as shown in Table 4.

\subsection{Effect of Yinzhihuang injection on agar-induced granulation tissue}

The inhibition rate of toe swelling in the $0.02 \mathrm{mg} / \mathrm{kg}$ Yinzhihuang group was $22.39 \%$. There was no significant difference between the $0.02 \mathrm{~g} / \mathrm{kg}$ Yinzhihuang group and the sodium salicylate group $(p>0.05)$, as shown in Table 5).

\subsection{Effect of Yinzhihuang injection on nitric oxide quantification of LPS-induced peritoneal macrophages}

The content of NO released into the supernatant of mouse peritoneal macrophages cultured with LPS was significantly reduced by the addition of the Yinzhihuang injection at a dose of 0.04 and 0.4 $\mathrm{mg} / \mathrm{ml}$, incomparison to the control group In each group, the NO content was significantly elevated in

Table 4

Effect of Yinzhihuang injection on swelling of rat toe induced by egg albumen

\begin{tabular}{|c|c|c|c|c|c|c|}
\hline Group & Drug & Dosage $(\mathrm{g} / \mathrm{kg})$ & Volume $(\mathrm{ml} / \mathrm{kg})$ & Dose (ml/kg) & Swelling of toe $(\mathrm{ml})$ & Inhibition rate $(\%)$ \\
\hline 1 & Yinzhihuang & 0.04 & 2.5 & 2.5 & $1.463 \pm 0.085 * * \#$ & 22.30 \\
\hline 2 & Yinzhihuang & 0.02 & 2.5 & 2.5 & $1.487 \pm 0.030$ **\# & 21.03 \\
\hline 3 & Yinzhihuang & 0.01 & 2.5 & 2.5 & $1.663 \pm 0.048 * \&$ & 11.68 \\
\hline 4 & Yinzhihuang & 0 & 2.5 & 2.5 & $1.883 \pm 0.165 \# \& \&$ & 0 \\
\hline 5 & $2 \% \mathrm{NaSA}$ & 0.5 & 2.5 & 2.5 & $1.512 \pm 0.079 *$ & 19.39 \\
\hline
\end{tabular}

$\& p<0.05 \mathrm{vs}$. Yinzhihuang $(0.04 \mathrm{~g} / \mathrm{kg})$.

Table 5

Effect of Yinzhihuang injection on mouse weight of agar-induced granulation tissue

\begin{tabular}{|c|c|c|c|c|c|}
\hline Group & Drug & $\begin{array}{l}\text { Dosage } \\
(\mathrm{g} / \mathrm{kg})\end{array}$ & $\begin{array}{l}\text { Volume } \quad(\mathrm{ml} / 10 \\
\mathrm{g})\end{array}$ & $\begin{array}{l}\text { General average of adding weight } \\
(\mathrm{g})\end{array}$ & $\begin{array}{l}\text { Inhibition } \\
(\%)\end{array}$ \\
\hline 1 & Yinzhihuang & 0.04 & 0.2 & $0.341 \pm 0.055 * *$ & 22.39 \\
\hline 2 & Yinzhihuang & 0.02 & 0.2 & $0.389 \pm 0.032 *$ & 11.39 \\
\hline 3 & Yinzhihuang & 0.01 & 0.2 & $0.420 \pm 0.025 \# \&$ & 4.33 \\
\hline 4 & Yinzhihuang & 0 & 0.2 & $0.439 \pm 0.057 \# \&$ & 0 \\
\hline 5 & $2 \% \mathrm{NaSA}$ & 0.2 & 0.2 & $0.358 \pm 0.042 *$ & 18.40 \\
\hline
\end{tabular}




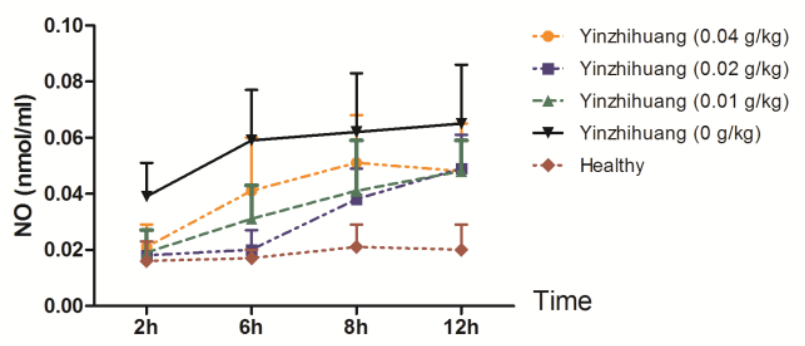

Fig. 2. Effect of Yinzhihuang injection on NO quantification of LPS-induced peritoneal macrophages from BALB/c mice. The peritoneal macrophages were obtained from BALB/c mice on day four post injection of $4 \%$ thioglycolate. $2-5 \times 10^{5}$ cells were cultured in RPMI 1640 medium in 96 -well culture plates at $37^{\circ} \mathrm{C}$ under $5 \% \mathrm{CO}_{2}$ atmosphere. Group I was treated with $0.04 \mathrm{~g} / \mathrm{kg}$ Yinzhihuang and $1 \mu \mathrm{g} / \mathrm{ml} \mathrm{LPS}$; group II was treated with $0.02 \mathrm{~g} / \mathrm{kg}$ Yinzhihuang; and group III was treated with $0.01 \mathrm{~g} / \mathrm{kg}$ Yinzhihuang and $1 \mu \mathrm{g} / \mathrm{mL}$ LPS. Group IV was treated with PBS. Model group IV was treated with $1 \mu \mathrm{g} / \mathrm{ml} \mathrm{LPS.}$ The level of nitric oxide, as measured by nitrite production 2, 6, 8, and 12 hours after infection, was determined using the Griess reagent (Invitrogen). The absorbance at $550 \mathrm{~nm}$ was measured by UV spectrophotometer.

12 hours, and decreased in 24 hours due to magrophage death. The drug groups showed significantly lower NO content than the control group in 2, 6, 8, and 12 hours, as shown in Figure 2.

\section{Discussion}

Yinzhihuang injection, a compound used in traditional Chinese medicine, has been widely used for its biological efficacy and low toxicity. The flavonoid baicalin is the main component of the Yinzhihuang injection, and has various pharmacological effects. While investigating a cure for infectious serositis, we observed the effects of Yinzhihuang injection treatments on RA-induced infectious serositis in ducks. We explored the link between anti-inflammatory effects of Yinzhihuang injection in vivo and antibacterial effects in vitro. Yinzhihuang injection was able to effectively treat infectious serositis in ducks. Additionally, it increased SOD activity, and decreased the concentrations of MDA and NO in RA-induced 16-day-old ducks. This study showed that SOD activity decreased, iNOS increased, and MDA content increased in the serum of ducks suffering from RA-induced infectious serositis. All these factors may weaken the antioxidant system of the ducks, which will subject most of the cell and tissue structures of the duck to different degrees of peroxidation damage. Simultaneously, extensive inflammation appeared in ducks with RA, eventually leading to death. The cure rate of the $0.02 \mathrm{~g} / \mathrm{kg}$ Yinzhihuang injection was $80 \%$ after 36 hours of RA infection. We explored whether baicalin worked to restrain RA growth directly, or if its curative properties are derived from antioxidant effects against RA-induced oxidant [32].

While determining the inhibition zone of Yinzhihuang injection against RA by the Oxford Cup method, it was demonstrated that Yinzhihuang injection could inhibit RA growth. RA was very sensitive in the $0.04 \mathrm{~g} / \mathrm{ml}$ Yinzhihuang. We found that the effect of Yinzhihuang injection on active and chronic inflammation is conspicuous through three inflammatory models. In a xylene-induced mouse auricle swelling test, the swelling inhibition rate of $0.04 \mathrm{~g} / \mathrm{kg}$ Yinzhihuang injection was $5.21 \%$. In egg albumen-induced rat postpeds metatarsus swelling test, revealed that 0.04 and $0.02 \mathrm{~g} / \mathrm{kg}$ Yinzhihuang injections had an anti-inflammatory effect on egg albumen-induced swelling, with swelling inhibition rates of $22.30 \%$ and $21.03 \%$, respectively. In the agar-induced granulation tissue test in mice, $0.04 \mathrm{~g} / \mathrm{kg}$ Yinzhihuang injection was able to effectively inhibit granulation tissue caused 
by agar.

Mouse peritoneal macrophages were stimulated with LPS, which induced dynamic effects on macrophage secretion of NO at different concentrations of Yinzhihuang injection. Results demonstrate that the amount of NO in macrophages and Yinzhihuang injection concentrations was dose-dependent, while the amount of NO in macrophages was time-dependent.

In conclusion, Yinzhihuang injections could promote NO and MDA content, as well as the activity of NOS in the serum of ducks with RAIIS. Is also positively inhibits the inflammatory response in mice or rats.

\section{Acknowledgments}

This work has been supported by Special Fund for Agro-scientific Research in the Public Interest with the grant number 201303040 and Science and Technology Projects of China with the grant number 2013FY110600.We thank Southwest University Rongchang Campus Preventive Medicine laboratory for their technical aid.

\section{References}

[1] P. Cavalcante, L. Maggi, L. Colleoni, R. Caldara, T. Motta, C. Giardina, C. Antozzi, S. Berrih-Aknin, P. Be rnasconi and R. Mantegazza, Inflammation and epstein-barr virus infection are common features of myasthenia gravis thymus: Possible roles in pathogenesis, Autoimmune Diseases 2011 (2011), 213092.

[2] Z. He, J. Ke, X. He, L. Lian, L. Sun, Z. Chen, X. Wu and P. Lan, Inflammation promotes the development of colitis-associated colorectal cancer, Chinese Journal of Gastrointestinal Surgery 17 (2014), 706-710.

[3] H.J. Ko, S.K. Oh, J.H. Jin, K.H. Son and H.P. Kim, Inhibition of experimental systemic inflammation (septic inflammation) and chronic bronchitis by new phytoformula BL containing broussonetia papyrifera and lonicera japonica, Biomolecules \& Therapeutics 21 (2013), 66-71.

[4] S.de Oliveira, A. Lopez-Munoz, S.P. Candel, Pelegrin, A. Calado and V. Mulero, ATP modulates acute inflammation in vivo through dual oxidase 1-derived $\mathrm{H}_{2} \mathrm{O}_{2}$ production and NF-kappaB activation, The Journal of Immunology 192 (2014), 5710-5719.

[5] D. Tsounis, G. Bouras, G. Giannopoulos, C. Papadimitriou, D. Alexopoulos and S. Deftereos, Inflammation markers in essential hypertension, Medicinal Chemistry 10 (2014), 672-681.

[6] S.J. Sasindran and J.B Torrelles, Mycobacterium tuberculosis infection and inflammation: What is beneficial for the host and for the bacterium? Frontiers in Microbiology 2 (2011), 2.

[7] S. Vairo, V. Saey, C. Bombardi, R. Ducatelle and H. Nauwynck, The recent european isolate (08P178) of equine arteritis virus causes inflammation but not arteritis in experimentally infected ponies, Journal of Comparative Pathology 151 (2014), 238-243.

[8] J.E. Goodman, L.J. Hofseth, S.P Hussain and C.C. Harris, Nitric oxide and p53 in cancer-prone chronic inflammation and oxyradical overload disease, Environmental and Molecular Mutagenesis 44 (2004), 3-9.

[9] G.M. Bodienkova, R. Alekseev, E. Boklazhenko and S. Kurchevenko, Inflammation mediators in employees in chronic exposure to neurotoxicants, International Journal of Occupational Medicine and Environmental Health 27 (2014), 619-626.

[10] A. Farrell, Immunology: Spreading inflammation, Nature Medicine 20 (2014), 813.

[11] M. Uddin and V.A. Diwadkar, Inflammation and psychopathology: What we now know, and what we need to know, Social Psychiatry and Psychiatric Epidemiology 49 (2014), 1537-1539.

[12] C.J. Hawkey, N.J. Talley, J.M. Scheiman, R.H. Jones, G. Langstrom, J. Naesdal and N.D. Yeomans, Maintenance treatment with esomeprazole following initial relief of non-steroidal anti-inflammatory drug-associated upper gastrointestinal symptoms: The NASA2 and SPACE2 studies, Arthritis Research and Therapy 9 (2007), 17.

[13] E. Khodaverdi, R. Honarmandi, M. Alibolandi, R.R. Baygi, F. Hadizadeh and G. Zohuri, Evaluation of synthetic zeolites as oral delivery vehicle for anti-inflammatory drugs, Iranian Journal of Basic Medical Sciences 17 (2014), 337-343.

[14] M.V. Aun, M. Blanca, L.S. Garro, M.R. Ribeiro, J. Kalil, A.A. Motta, M. Castells and P. Giavina-Bianchi, Nonsteroidal 
anti-inflammatory drugs are major causes of drug-induced anaphylaxis, The Journal of Allergy and Clinical Immunology : In Practice 24 (2014), 414-420.

[15] N. Blanca-Lopez, I. Dona, J.R. Perkins, G. Canto, J.A. Cornejo-Garcia and M. Blanca, Multiple nonsteroidal anti-inflammatory drug-induced cutaneous disease: Relevance, natural evolution and relationship with atopy, International Archives of Allergy and Immunology 164 (2014), 147-148.

[16] J.H. Kim, S.H. Park, C.S. Cho, S.T. Lee, W.H. Yoo, S.K. Kim, Y.M. Kang, J.S. Rew, Y.W. Park, S.K. Lee, Y.C. Lee, W. Park and D.H. Lee, Preventive efficacy and safety of rebamipide in nonsteroidal anti-inflammatory drug-induced mucosal toxicity, Gut and Liver 8 (2014), 371-379.

[17] R.J. Woodman, Quantifying nonsteroidal anti-inflammatory drug (NSAID) risk: Can combining the evidence with the use of a network meta-analysis provide a solution? Therapeutic Advances in Drug Safety 2 (2011), 137-139.

[18] W. Ding, J. Gu, L. Cao, N. Li, G. Ding, Z. Wang, L. Chen, X. Xu and W. Xiao, Traditional Chinese herbs as chemical resource library for drug discovery of anti-infective and anti-inflammatory, Journal of Ethnopharmacology 155 (2014), $589-598$.

[19] L. Li, W.Q. Zhang, F.X. Li and B. Jiang, The efficacy of polyene phosphatidylcholine and Yinzhihuang injection treatment for the intrahepatic cholestasis of chronic viral hepatitis, The Journal of Medical Theory and Practice 24 (2011), 1126-1127.

[20] N. Liu, J. Xu, H. Li and S.C. He. Protective effects of Yinzhihuang injection on bilirubin-induced brain injury in neonatal rat, Journal of Jinan University 32 (2011), 589-592.

[21] M. Lin, L. Li, Y. Zhang, L. Zheng, M. Xu, R. Rong and T. Zhu, Baicalin ameliorates $\mathrm{H}_{2} \mathrm{O}_{2}$ induced cytotoxicity in HK-2 cells through the inhibition of ER stress and the activation of Nrf2 signaling, International Journal of Molecular Sciences 15 (2014), 12507-12522.

[22] H. Wang, H. Wang, S. Huang, H. Zhao, Y. Cao, G. Wang, D. Wang, Z. Wang and Y. Liu, Inhibitory effect of baicalin on collagen-induced arthritis in rats through the nuclear factor-kappaB pathway, Journal of Pharmacology and Experimental Therapeutics 350 (2014), 435-443.

[23] S. Yang, Y. Fu, X. Wu, Z. Zhou, J. Xu, X. Zeng, N. Kuang and Y. Zeng, Baicalin prevents Candida albicans infections via increasing its apoptosis rate, Biochemical and Biophysical Research Communications 451 (2014), 36-41.

[24] W. Zheng, F. Wang, X. Cao, H. Pan, X. Liu, X. Hu and Y. Sun, Baicalin protects PC-12 cells from oxidative stress induced by hydrogen peroxide via anti-apoptotic effects, Brain Injury 28 (2014), 227-234.

[25] Y. Ding, J. Dou, Z. Teng, J. Yu, T. Wang, N. Lu, H. Wang and C. Zhou, Antiviral activity of baicalin against influenza A (H1N1/H3N2) virus in cell culture and in mice and its inhibition of neuraminidase, Archives of Virology 159 (2014), 3269-3278.

[26] W. Lee, S.K. Ku and J.S. Bae, Antiplatelet, anticoagulant and profibrinolytic activities of baicalin, Archives of Pharmacal Research 38 (2015), 893-903.

[27] P.F. Liu, F.G. Han, B.B Duan, T.S. Deng, X.L. Huo, and M.Q. Zhao, Purification and antioxidant activities of baicalin isolated from the root of huangqin (Scutellaria baicalensis gcorsi), Journal of Food Science and Technology 50 (2013), 615-619.

[28] M. Guo, N.S. Zhang, D. Li, D. Liang, Z. Liu, F. Li, Y. Fu, Y. Cao, X. Deng and Z. Yang, Baicalin plays an anti-inflammatory role through reducing nuclear factor-kappaB and p38 phosphorylation in $\mathrm{S}$. aureus-induced mastitis, International Immunopharmacology 16 (2013), 125-130.

[29] J. Zhu, J. Wang, Y. Sheng, Y. Zou, L. Bo, F. Wang, J. Lou, X. Fan, R. Bao, Y. Wu, F. Chen, X. Deng and J. Li, Baicalin improves survival in a murine model of polymicrobial sepsis via suppressing inflammatory response and lymphocyte apoptosis, PloS One 7 (2012), e35523.

[30] S.Y. Xu, R.L. Bian and X. Chen, Pharmacological Experiment Methodology, People's Medical Publishing House, Beijing, 2002, pp. 1647-1719.

[31] A.B. Carneiro, B.M. Iaciura, L.L. Nohara, C.D. Lopes, E.M. Veas, V.S. Mariano, P.T. Bozza, U.G. Lopes, G.C. Atella, I.C. Almeida and M.A. Silva-Neto, Lysophosphatidylcholine triggers TLR2- and TLR4-mediated signaling pathways but counteracts LPS-induced NO synthesis in peritoneal macrophages by inhibiting NF-kappaB translocation and MAPK/ERK phosphorylation, PloS One 8 (2013), e76233.

[32] E. Moghaddam, B.T. Teoh, S.S. Sam, R. Lani, P. Hassandarvish, Z. Chik, A. Yueh, S. Abubakar and K. Zandi, Baicalin, a metabolite of baicalein with antiviral activity against dengue virus, Scientific Reports 4 (2014), 5452. 